Original Article

\title{
Single hook model of fiber figures in card webs
}

\begin{abstract}
In card webs, many fibers are not straight and generally have hooks or bends. In this study, a onedimensional fiber figure model is discussed. Using correlation coefficients, a simple classification method for fiber figure is proposed. This method enables us to classify fiber figures into five categories, straight, leading- and trailing-hooked, and leading- and trailing-bent figures. This method is then applied to experimental data. The results indicate that the absolute values of the correlation coefficients of the vector quantities between the measured fiber figures and one of our models are greater than 0.8 in many cases. Thus, our proposed method is viable for the classification of fiber figures in card webs.
\end{abstract}

\section{Key words}

correlation coefficients of vector quantities, card web, fiber figure

During carding, fibers in card webs tend to be stretched in the machine direction; however, windings are often involved with the fibers. Carding is an important process that influences fiber condition in card webs; thus many researchers have investigated in fiber condition in card webs ${ }^{1-4}$. Wakankar et.al. studied the relationship between carding parameters and hook formation ${ }^{5}$. Ishtiaque et.al. studied the effect of carding and drawing conditions on fiber hook in spun yarns ${ }^{6}$. For one more example, Wagle and Govindarajulu studied the effect of drafting on fiber hook removal ${ }^{7}$. In their study, they defined U shaped and ordinary hooks, and observed that drafting effects were different depend on the types of hooks. Recently, Miao and Glassey studied this topic with card webs prepared for needle-punched nonwovens ${ }^{8}$. In their study, they classified fiber figures into five types; hooks, $U$ bends, straight fibers, loops, and entanglements; however, this classification performed by the naked eye. Thus, e.g., clearly defining the boundary between hooks and $U$ bends was difficult.

In the present study, we introduce a method to classify fiber figures based on data analysis. In particular, we discuss the classification of fiber figures into five types; straight, leading- and trailinghooked, and leading- and trailing-bent fibers, which are dominant in card webs.

\section{Theoretical discussion}

\section{Correlation coefficients}


Suppose we have two sets of variables, $\left\{u_{k}\right\}$ and $\left\{v_{k}\right\}$. A correlation coefficient is used to express the similarity of their variation state. When the averages of these variables are zero, the correlation coefficient is defined as follows:

$$
r=\frac{\sum u_{k} v_{k}}{\sqrt{\sum u_{k}^{2}} \sqrt{\sum v_{k}^{2}}}
$$

If the variables are vector quantities, the correlation coefficient is defined using a scalar product of the variables as follows:

$$
r=\frac{\sum u_{k} \cdot v_{k}}{\sqrt{\sum\left|u_{k}\right|^{2}} \sqrt{\sum\left|v_{k}\right|^{2}}} .
$$

\section{Straight line segments}

First, to discuss a fiber figure in card webs, we focus on whether the fiber is straight, i.e., whether the correlation coefficient between a straight line segment and the fiber's figure is high. Thus, we define a straight line segment as the first typical fiber figure. We assume that this segment is a part of the $x$ axis, starting from $x=-0.5$ and ending at $x=0.5$; therefore, it has unit length, and the average of its coordinate values is zero. In this manner, we also assume its direction from -0.5 to 0.5 as the positive direction; if its direction is opposite, we call it an anti-straight line segment.

\section{Bent figures}

As the second model figure, we assume a completely folded line segment at the midpoint. If the fold appears on the right side, we call it a leading-bent figure. If the fold appears on the other side, we call it a trailing-bent figure.

\section{Hooked figures}

We introduce the third model figure, hooked figures, as a more general type. In this case, a line segment of unit length is folded in a location other than the midpoint. Now, we call the folded point a hook point. Figure 1 shows three model figures. In this study, we discuss fiber figures that only have one hook point. Similar to bent figure cases, there are two types of hooked figures, called leading- and trailing-hooked figures. 


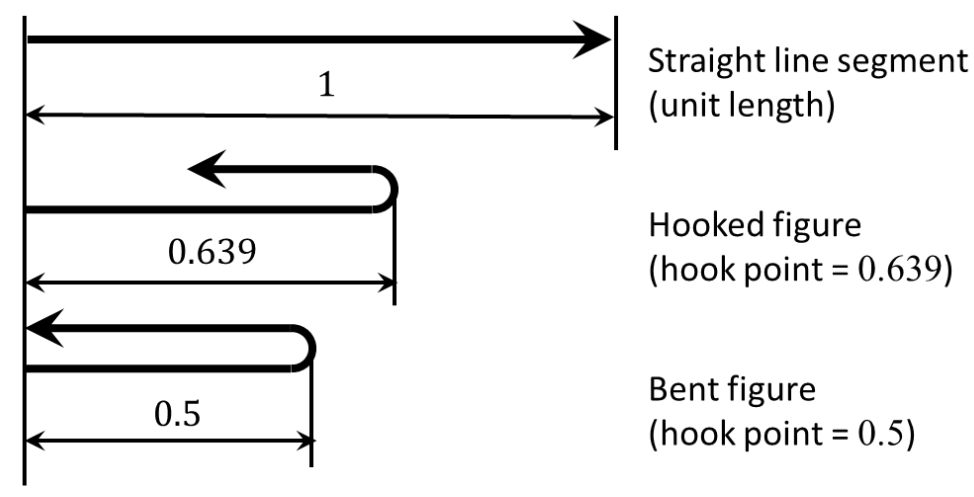

Figure 1. Three model figures.

\section{Mathematical expressions of model figures}

In this subsection, we discuss the mathematical expressions of aforementioned model figures.

\section{Straight line segments}

A straight line segment is the simplest figure. Suppose a one-dimensional coordinate system. To fit the center of the figure to the origin of coordinate, we assume that the starting point is -0.5 . Using a parameter $t$, the straight line segment can be expressed as follows:

$$
\begin{gathered}
x=t-\frac{1}{2} \\
(0 \leq t \leq 1) .
\end{gathered}
$$

An anti-straight line segment can be expressed as follows:

$$
\begin{aligned}
& x=-t+\frac{1}{2} \\
& (0 \leq t \leq 1) .
\end{aligned}
$$

\section{Bent figures}

In this case, as these figures are folded at the midpoint, we need two equations to express these figures. A leading-bent figure is expressed as follows:

$$
\left\{\begin{array}{cc}
x=t-\frac{1}{4} & \left(0 \leq t \leq \frac{1}{2}\right) \\
x=-t+\frac{3}{4} & \left(\frac{1}{2} \leq t \leq 1\right)
\end{array} .\right.
$$


Similarly, a trailing-bent figure can be expressed as follows:

$$
\left\{\begin{array}{cc}
x=-t+\frac{1}{4} & \left(0 \leq t \leq \frac{1}{2}\right) \\
x=t-\frac{3}{4} & \left(\frac{1}{2} \leq t \leq 1\right)
\end{array} .\right.
$$

Notably, leading- and trailing-bent figures are identical; however the signs in the right sides of the equations are inverted. These figures are also shifted to fit the centers to the origin of the coordinates.

\section{Hooked figures}

Intuitively determining the centers of hooked figures may appear difficult. In these cases, we select the gravity centers for the centers of these figures. We derive the expressions for the leading-hooked figure to fit the gravity centers to the coordinate origin as follows:

$$
\left\{\begin{array}{cc}
x=t+h^{2}-2 h+\frac{1}{2} & (0 \leq t \leq h) \\
x=-t+h^{2}+\frac{1}{2} & (h \leq t \leq 1)
\end{array},\right.
$$

where $h$ is the hook point. We omit the expressions of the trailing-hooked figures, which are obtained by inverting all the signs of Equation (7).

\section{Relation among model figures}

The above expressions clarify the relations among the corresponding figures. For example, a leadingbent figure is identical to a leading-hooked figure of $h=0.5$. Furthermore, straight line segment is expressed by the leading-hooked figure of $h=1$. Similar relations are observed among the trailinghooked figure, trailing-bent figure, and anti-straight line segments.

Now, supposing a leading-hooked figure of $h=0$, the figure changes its direction at the starting point; thus resulting in an anti-straight line segment. In the same manner, a trailing-hooked figure can be related to a straight line segment.

\section{Correlation coefficients among model figures}

Clearly, the correlation coefficients between identical figures are unity, and for opposite figures, e.g., the case of a straight line segment and an anti-straight line segment, a negative correlation coefficient is obtained. In this subsection, we discuss the correlation coefficients corresponding to other combinations of model figures. For this purpose, we define the correlation coefficients between 
functions. By substituting the summations of Equation (1) with integrals, we satisfy the above purpose, as follows:

$$
r=\frac{\int u(t) v(t) d t}{\sqrt{\int u(t)^{2} d t} \sqrt{\int v(t)^{2} d t}} .
$$

\section{Correlation coefficients between straight line segments and bent figures}

In this case, we concentrate on the numerator of Equation (8). Note that as bent figures are expressed by two equations, we need to divide the interval of integration into two parts. For example, the numerator for a straight line segment and leading-bent figure results in

$$
\int_{0}^{\frac{1}{2}}\left(t-\frac{1}{2}\right)\left(t-\frac{1}{4}\right) d t+\int_{\frac{1}{2}}^{1}\left(t-\frac{1}{2}\right)\left(-t+\frac{3}{4}\right) d t=0
$$

As observed in Equation (9), because the numerator of Equation (8) is zero, the correlation coefficient results in zero irrespectively of the denominator. Similar results can be obtained from other combinations, e.g., anti-straight and a trailing-bent figures.

\section{Correlation coefficients between straight line segments and hooked figures}

Next, we present the case of a straight line segment and leading-hooked figure. In this case, the integral interval of the numerator in Equation (8) is divided at $t=h$. We obtained the result as follows:

$$
r_{s}=\frac{-\frac{1}{3} h^{3}+\frac{1}{2} h^{2}-\frac{1}{12}}{\sqrt{\frac{1}{12}} \sqrt{-h^{4}+2 h^{3}-h^{2}+\frac{1}{12}}} .
$$

In the combinations of an anti-straight line segment and leading-hooked figure or a straight line segment and trailing-hooked figure, the signs of the numerator in Equation (10) are inverted. In the combination of an anti-straight line segment and trailing-hooked figure, Equation (10) can be used.

\section{Correlation coefficients between bent and hooked figures}

Here, we discuss the combination of bent and hooked figures. In this case, the integrand of the numerator of Equation (8) changes two times along the integral interval; i.e., at $t=0.5$, the 
expression for bent figures changes, and at $t=h$, that for hooked figures changes. Therefore, we divide the interval of the integral into three parts. It is emphasized that, the method of division depends on the value of $h$ (i.e., whether it is greater or smaller than 0.5 ). If $h>0.5$, we obtain the result as follows:

$$
r_{b}=\frac{\frac{1}{3} h^{3}-\frac{3}{4} h^{2}+\frac{1}{2} h-\frac{1}{12}}{\sqrt{\frac{1}{48}} \sqrt{-h^{4}+2 h^{3}-h^{2}+\frac{1}{12}}} .
$$

If $h<0.5$, we obtain

$$
r_{b}=\frac{-\frac{1}{3} h^{3}+\frac{1}{4} h^{2}}{\sqrt{\frac{1}{48}} \sqrt{-h^{4}+2 h^{3}-h^{2}+\frac{1}{12}}} .
$$

\section{Correlation coefficients between two hooked figures}

Finally, we extend this result to the most general case of two hooked figures. The results are as follows:

$$
r=\frac{\frac{1}{3} h_{2}^{3}-h_{1}^{2} h_{2}^{2}-\frac{1}{2} h_{2}^{2}+2 h_{1}^{2} h_{2}-\frac{1}{3} h_{1}^{3}-\frac{1}{2} h_{1}^{2}+\frac{1}{12}}{\sqrt{-h_{1}^{4}+2 h_{1}^{3}-h_{1}^{2}+\frac{1}{12}} \sqrt{-h_{2}^{4}+2 h_{2}^{3}-h_{2}^{2}+\frac{1}{12}}} \quad\left(h_{1} \leq h_{2}\right)
$$

and

$$
r=\frac{-\frac{1}{3} h_{2}^{3}-h_{1}^{2} h_{2}^{2}-\frac{1}{2} h_{2}^{2}+2 h_{1} h_{2}^{2}+\frac{4}{3} h_{1}^{3}-\frac{1}{2} h_{1}^{2}+\frac{1}{12}}{\sqrt{-h_{1}^{4}+2 h_{1}^{3}-h_{1}^{2}+\frac{1}{12}} \sqrt{-h_{2}^{4}+2 h_{2}^{3}-h_{2}^{2}+\frac{1}{12}}} \quad\left(h_{1} \geq h_{2}\right)
$$

where $h_{1}$ and $h_{2}$ are the hook points of the figures.

\section{Correlation functions of hook points}

We regard Equations (10), (11), and (12) as functions of the hook point $h$. Thus, we plotted graphs of the functions in Figure 2, where $r_{s}$ increases monotonically with an increase of $h$. In contrast, $r_{b}$ increases with an increase of $h$ up to $h=0.5$, decreases thereafter. 


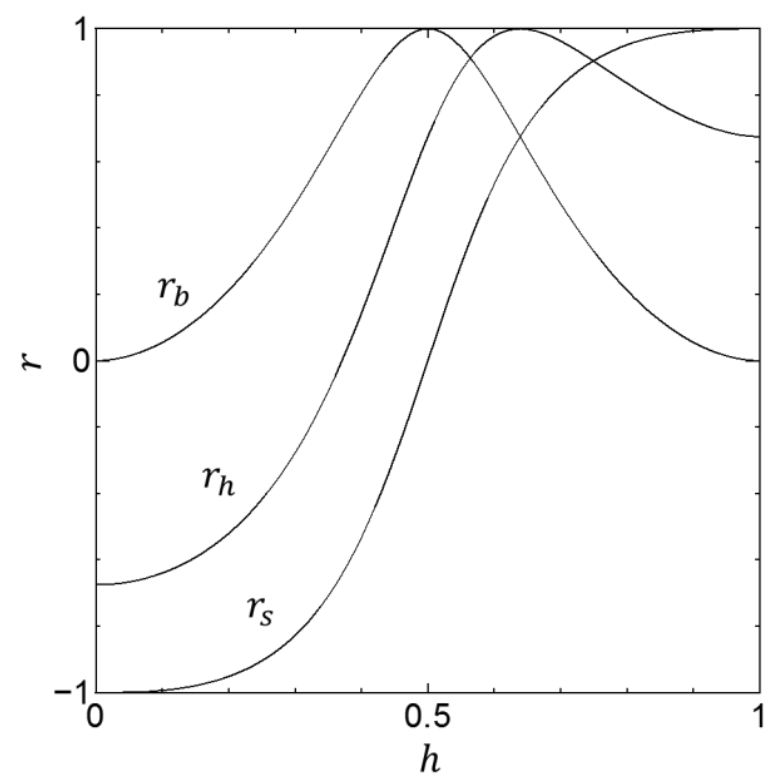

Figure 2. Correlation coefficients as functions of hook point (Leading hooks).

In Figure 2, the curves corresponding to $r_{s}$ and $r_{b}$ intersect at $h \cong 0.639$. In the range that $h>$ 0.639 , it results in $r_{s}>r_{b}$, namely, the correlation coefficient of such hooked figure with straight line segment is greater than that with bent figure, and vice versa. Thus, with this basis, we can classify the figures into two types; straight line segments and bent figures. However, investigating the actual fiber's figure appears to be very difficult. Notably, the correlation coefficient value of the intersection point is only $r \cong 0.675$ for both categories which is very small for our objective.

\section{The most typical hooked figure}

In the previous subsection, the result that the correlation coefficients $r_{s}$ and $r_{b}$ near $h=0.639$ were very small was an issue. Therefore, in this subsection, we change our viewpoint. As the hooked figure of $h=0.639$ is not similar to either straight line segments or bent figures, we regard it as the most typical hooked figure. Recalling Figure 1, there are three model figures. The last is a bent figure corresponding to $h=0.5$, and the first is a straight line segment of $h=1$. The case of $h=0.639$ is indicated between them in the figure. Recalling Figure 2, we also plotted the curve corresponding to the model hook as $r_{h}$ using Equation (11). This curve intersects the curve of $r_{b}$ at $h \cong 0.564$ and $r_{s}$ at $h \cong 0.749$. By selecting these intersection points as criteria of classification, we categorize any hooked figure of $0.5 \leq h \leq 1$ into three categories, straight line segment, hooked and bent figures. As the correlation coefficients of the intersection points are greater than 0.9; any hooked figures of $0.5 \leq h \leq 1$ satisfy $r>0.9$ by adequate classification. We also obtained similar results in the range of $0 \leq h \leq 0.5$. 


\section{Hook points of actual data}

To apply our method to actual fibers, we needed hook points of measured data. Equation (2) was used for this purpose. First, we calculated the main direction of the data using the maximum variance method reported in a previous study ${ }^{9}$. Then, we fitted the model direction to the fiber direction. The points on the fiber corresponded to $\left\{u_{k}\right\}$, and those on a hooked figure model corresponded to $\left\{v_{k}\right\}$ in Equation (2). Then we calculated the correlation coefficient using Equation (2) by changing the hook point $h$ and selected the value of $h$ that maximized the absolute value of the correlation coefficient as the hook point of the measured data. In addition, based on the value of the hook points and the criteria mentioned in the previous subsection, we categorized the fibers into three types: bent, hooked, or straight fibers. Furthermore, the signs of the correlation coefficients indicate the direction of the figures. Thus, we have six categories: straight- and anti-straight line segments, leading- and trailing hooks, and leading- and trailing-bends; however, there is no meaning to distinguish the directions of straight fibers. Thus, we should unite the straight- and anti-straight line segments into one category. Therefore, we have five categories for real fibers.

Using this method, any type of fiber figure can be classified; however, in some cases, the classification is not apposite. For example, an entangled fiber can also be classified into one of the abovementioned types; however, this practice appears to be unreasonable. Considering this point, we need to verify the maximized correlation coefficient before classification.

\section{Experiment}

The details of the experimental method are reported in a previous study ${ }^{9}$. Thus, a brief summary of our experiment is provided here.

We used polyester fibers (fiber length $=38 \mathrm{~mm}$, fiber fineness $=1.4 \mathrm{dtex}$ ) as the raw material for our experiment. As tracer fibers, $0.1 \%$ colored fibers were added and then processed by a miniature flat card. Thin sheets of produced card webs were laminated between transparent polyester films. Then, we captured 99 images of tracer fibers of laminated sheets with an image scanner of 4800 dpi and 8bit gray scale mode. From the image data, we resampled equal-interval polyline with 3000 vertices. Using the coordinate values of the vertices, we derived the hook points of the fibers. In the calculation, the solver feature of Microsoft Excel was used to determine the hook point values of the fiber data. Then, the obtained hook points and maximized correlation coefficients were plotted in a graph.

\section{Results and discussion}

\section{Scanned image and measured hook points}

Figure 3 shows an example of scanned fiber image in card webs. Such images were processed to obtain 
fiber figure data.

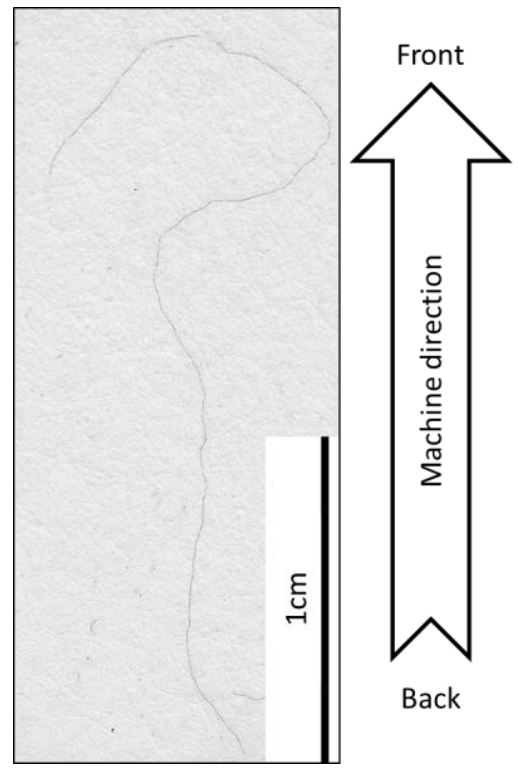

Figure 3. An example of scanned image.

Figure 4 shows examples of the fiber figures in card webs. We also drew model figures that maximize the absolute values of correlation coefficient with the corresponding measured data. The maximized values of correlation coefficient and corresponding hook point values are also indicated in Figure 4. As observed in the figure, in some cases, describing the fiber's figure using our model is difficult; however, in many cases, the fiber figures are well summarized using our single hook model.
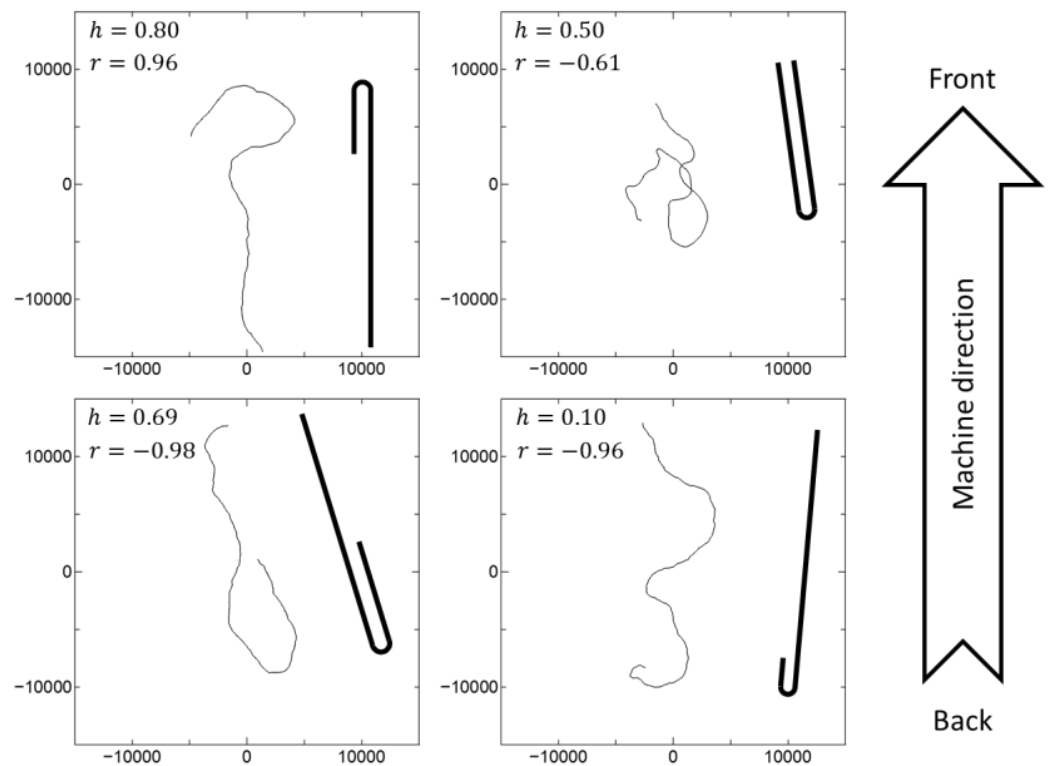

Back

Figure 4. Obtained fiber images and most similar models (Unit: $\mu \mathrm{m})$. 


\section{Maximized correlation coefficients}

Figure 5 presents a plot of the maximized correlation coefficients and hook points of the measured data. The horizontal axis corresponds to hook point value and vertical axis corresponds to the maximized correlation coefficient value for each fiber figure data. The upper side of the graph corresponds to leading figures, and the lower side corresponds to trailing figures. The vertical dotted lines shown in the figure correspond to the boundaries of the fiber figure categories determined by the intersect points of curves in Figure 2. For example, at the intersect point of curves $r_{b}$ and $r_{s}$, the hook point $h=0.564$, thus we selected this value as the boundary of bent and hooked figures. That of hooked figure and straight line segment also decided as $h=0.749$ by the intersect point of $r_{h}$ and $r_{s}$ in Figure 2. Using these criteria, we can easily categorize a fiber's figure based on the hook points. In Figure 5, we have two leading hooked figure regions along the upper side of the graph. The difference between these regions is caused by the numbering order of the measured data; thus, there is no essential difference between them for real fibers, and thus we consider them as one category. Similarly, we treat two regions corresponding to the trailing-hooked figure located in the lower side of the graph as one category. Furthermore, the two straight regions located at the upper corners of the graph correspond to straight line segments and those located at the lower corners correspond to antistraight line segments. However, for real fiber, these segments are undistinguishable. Therefore we regard these four corners as one category.

Many plots are located near the upper and lower sides. Referring to the horizontal dotted lines in Figure 5, the absolute values of the correlation coefficient are greater than 0.8 in many cases. There are more points in the lower region than in the upper region, which indicates that the fibers in card webs tend to have trailing rather than leading figures. We classified the observed fibers according to this plot, and the result is summarized in Table 1.

Some points are located away from the upper and lower sides of the graph because the absolute values of their correlation coefficients with our models are small which indicates that summarizing the fibers corresponding to the points is difficult by our simple single hook model. For these fibers, we require some additional models, e.g., hooks on both ends. However, many fibers are considered to be well categorized by our single hook model. 


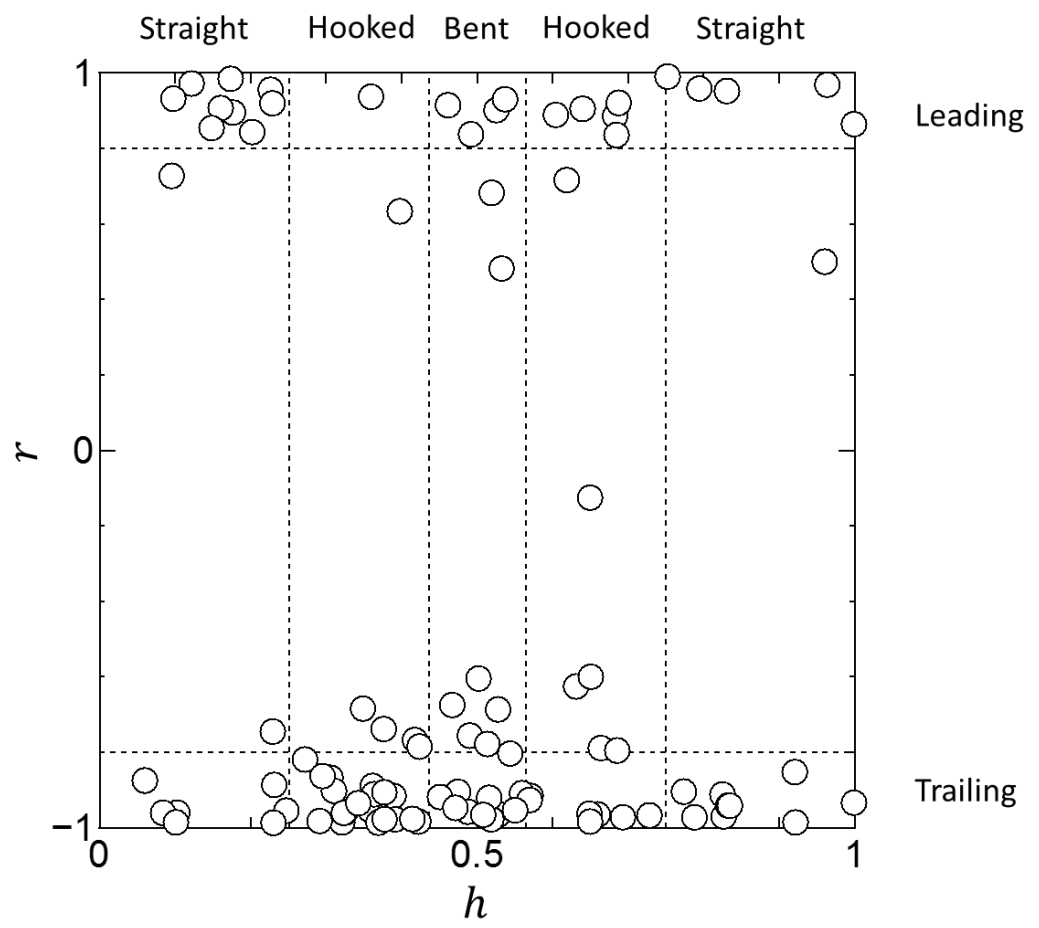

Figure 5. Maximized correlation coefficients and hook points of measured data.

Table 1. Results of classification.

\begin{tabular}{ccccccc}
\hline & Bent & Hooked & Straight & Unclassifiable & & Total \\
\hline Leading & 4 & 6 & - & - & & 10 \\
Trailing & 11 & 26 & - & - & & 37 \\
Undirectional & - & - & 30 & - & & 30 \\
Unclassifiable & - & - & - & 30 & 22 & \\
\hline Total & 15 & 32 & 30 & & 22 \\
\hline
\end{tabular}

\section{Conclusions}

We proposed a classification method for fiber figures in card webs based on correlation coefficients. Five dominant categories, i.e., straight, leading- and trailing-bent, and leading- and trailing-hooked figures were defined, and the criteria of classification were derived theoretically. The maximum correlation coefficient method which defines hook points of real fiber figures is also developed. To check the viability of proposed method, an experimental work was performed. The results showed that the hook points of real fiber figures were successfully determined using the maximum correlation coefficient method. Referring to the maximized correlation coefficient of each fiber figure, in some cases, the simple model proposed in this study was not sufficient; however, in many cases, the real fibers could be summarized using our single hook model. In addition, we can easily classify the fibers 
into five categories, based on the obtained hook points. Thus, it is confirmed that the method obtained by the theoretical discussion is viable to categorize fiber figures in card webs.

\section{Acknowledgement}

We should like thank to Mr. Shinya Sasaki for his assistance in the experiment.

\section{Funding}

This research received no specific grant from any funding agency in the public, commercial, or notfor-profit sectors.

\section{References}

1. Morton WE, Summers RJ. Fiber arrangement in card slivers. $J$ Textile Inst Proc 1949; 40(2): 106-116.

2. Garde AR, Wakankar VA, Bhaduri SN. Fiber configuration in sliver and roving and its effect on yarn quality. Textile Res $J 1961 ; 31(12)$ : 1026-1036.

3. Trott DW, Scardino FL. An investigation of fiber geometry in card webs. Textile Res J 1969; 39(11): 1031-1037.

4. Das D, Ishtiaque SM, Yadav S. Evaluation of fibre orientation in fibrewebs. Indian J Fibre Textile Res 2014; 39(1): 9-13.

5. Wakankar VA, Bhaduri SN, Ramaswamy BR, Ghosh GC. Some studies on the formation of hooks in carding. Textile Res $J$ 1961; 31(11): 931-940.

6. Ishtiaque SM, Mukhopadhyay A, Kumar A. Impact of carding parameters and draw frame speed on fibre axial distribution in ring-spun yarn. Indian J Fibre Textile Res 2009; 34(3): 231-238.

7. Wagle NP, Govindarajulu R. Some studies on the formation and removal of fiber hooks. Textile Res $J$ 1971; 41(5): 397-404.

8. Miao M, Glassey HE. An experimental study of the needled nonwoven process Part I: fiber geometry before needle punching. Textile Res $J$ 2004; 74(4): 329-332.

9. Sakaguchi A, Kimura H. Fiber orientation and aspect ratio calculation using image analysis. $J$ Textile Sci Eng 2015; 5(1): 187 1-3. 